\title{
Lifetime Maximization by Cooperative Sensor and Relay Deployment in Wireless Sensor Networks
}

\author{
Thanongsak Himsoon, W. Pam Siriwongpairat, Zhu Han, and K. J. Ray Liu \\ Department of Electrical and Computer Engineering and Institute for Systems Research \\ University of Maryland, College Park, MD 20742, USA.
}

\begin{abstract}
In this paper, cooperative sensor protocol and relay deployment in wireless sensor networks (WSNs) is proposed to improve the network lifetime. First, cooperative transmission is employed among sensor nodes. With an objective of maximizing the network lifetime under a constraint on bit-error-rate performance, we determine which sensors should cooperate and how much power to allocate for cooperation. A closed form solution is provided for a two-sensor WSN. Based on the obtained two-sensor solution, a fast suboptimal algorithm is developed for multiple sensor case. Moreover, the network lifetime is further improved by deploying cooperative relays in the network to help sensors transmit their information. The network lifetime is maximized by choosing the optimum location for each relay and optimally allocating the power that the relay helps each sensor. A suboptimal algorithm is proposed for the WSN with multiple relays. Simulation results show that the network lifetime of the proposed WSN with cooperative sensor employment improves 2.3 times compared with the non-cooperative WSN. In addition, deploying a cooperative relay in the proper location improves network lifetime up to 3 times of that for the non-cooperative WSN.
\end{abstract}

\section{INTRODUCTION}

In wireless sensor networks (WSNs), extending network lifetime is considered as an energy efficient technique that enables extensive uses of uninterrupted data gathering capability and alleviate the burden of replenishing batteries of all sensors. Several definitions of the network lifetime have been introduced (See [1], [2] and references therein), and they are suitable for different applications. One meaningful definition for missioncritical applications is the duration from the deployment of the network to the time that the battery of the first sensor is fully drained [1]. Several works based on this network lifetime definition have been proposed [2]-[6]. The problem of finding an energy-efficient tree to maximize network lifetime has been considered for broadcasting scenario [2]-[4] and multicasting scenario [5]. In addition, the static and dynamic maximum lifetime problems have been addressed in [2]-[5]. In [6], the network lifetime is maximized by employing the accumulative broadcast strategy which relies on the assumption that sensors cooperatively accumulate energy of unreliable receptions over the relay channels. The idea in [6] has been extended to maximize the network lifetime under multicasting scenario [7].

Recently, cooperation diversity paradigm [8]-[9] has been introduced to explore inherent spatial diversity through relay channels. Several cooperation protocols have been proposed, e.g. amplifyand-forward (AF) and decode-and-forward (DF) protocols. The exact performance analysis and optimum power allocation for the DF protocol have been investigated in [10]. The relevant works on which nodes are appropriate for cooperation and how much cooperation should be facilitated are provided in [11]-[12]. In [11], the performance is evaluated when the best relay is chosen according to average signal-to-noise ratio (SNR) level. In [12], the problem of who should cooperate and how much power should be allocated are addressed for multiuser OFDM networks. The proposed work in [13] considered the use of a set of relays employing AF protocol to extend the network lifetime in WSNs. However, the work focuses on physical layer, and cooperation among nodes have not been investigated.

In this paper, we propose to increase the network lifetime by exploiting the cooperation diversity in WSNs. The framework is based on the DF cooperation protocol; however, other cooperation protocols such as AF can be similar employed as well. We formulate an optimization problem to maximize the network lifetime under bit-error-rate (BER) and power constraints. First, we consider the cooperation among sensor nodes. We determine which sensors should cooperate and how much power to allocate for cooperative transmission. An analytical solution for a twosensor WSN is deduced to obtain some insights. In case of multisensor cooperative WSN, we propose a fast suboptimal algorithm to reduce the complexity of the formulated NP hard problem. Next, we further improve the network lifetime by deploying cooperative relays over a network of energy depleting sensors. We determine which locations to place the relays and how much power to cooperate. An efficient suboptimal algorithm is also developed. Simulation results are given to show network lifetime improvement over the non-cooperative WSN.

\section{System Model}

Consider a WSN with $N$ sensors, each randomly located in the network. The information collected by each sensor is transmitted via a route to its destination. Since routing is beyond the scope of this paper, we simply assume that the route for each sensor is already known and fixed during the lifetime of the network. In cooperative sensor network, each sensor can act as a source that sends information on its route, or a relay that helps forward information on other routes. For notation convenience, we denote $n_{j}$ as the next node of node $j(j=1,2, \ldots, N)$. The cooperative strategy comprises two phase. In Phase I, the source transmits information. Suppose sensor $j$ acts as the source and sensor $i$ acts as the relay, the received signal at the next node $n_{j}$ can be expressed as

$$
y_{j n_{j}}=\sqrt{P_{j j}} h_{j n_{j}} x_{j}+w_{j n_{j}},
$$

where $x_{j}$ denotes the symbol to be transmitted from sensor $j, P_{j j}$ is the transmitted power of sensor $j, h_{j n_{j}}$ is the fading coefficient from sensor $j$ to $n_{j}$, and $w_{j n_{j}}$ is additive noise. The channel coefficient $h_{j n_{j}}$ is modeled as a complex Gaussian random variable with zero mean and variance $\sigma_{j n_{j}}^{2}$, i.e., $\mathcal{C N}\left(0, \sigma_{j n_{j}}^{2}\right)$, and the noise $w_{j n_{j}}$ is $\mathcal{C N}\left(0, N_{0}\right)$ distributed. Taking into account the effects of path loss, the channel variance $\sigma_{j n_{j}}^{2}$ can be modeled as $\sigma_{j n_{j}}^{2}=k D_{j n_{j}}^{-\alpha}$, where $D_{j n_{j}}$ denotes distance between sensors $j$ and $n_{j}, \alpha$ is the propagation loss factor, and $k$ is a constant whose value depends on the propagation environment. The received 
signal at the relay (sensor $i$ ) is

$$
y_{j i}=\sqrt{P_{j j}} h_{j i} x_{j}+w_{j i},
$$

where $h_{j i}$ is the channel coefficient from node $j$ to node $i$, and $w_{j i}$ is an additive noise $\left(w_{j i} \sim \mathcal{C N}\left(0, N_{0}\right)\right)$. The channel $h_{j i}$ is $\mathcal{C N}\left(0, \sigma_{j i}^{2}\right)$ distributed with variance $\sigma_{j i}^{2}=k D_{j i}^{-\alpha}$, where $D_{j i}$ is the distance between sensor $j$ and sensor $i$.

In Phase II, the relay forwards the information only if the symbol is correctly decoded by which we assume for mathematical tractability that the relay can judge whether the decoded information is correct or not ${ }^{1}$. The received signal at node $n_{j}$ is

$$
y_{i n_{j}}=\sqrt{\tilde{P}_{i j}} h_{i n_{j}} x_{j}+w_{i n_{j}},
$$

where $\tilde{P}_{i j}=P_{i j}$ if the relay decodes the symbol correctly, otherwise $\tilde{P}_{i j}=0$. The channel coefficient $h_{i n_{j}}$ and the noise $w_{i n_{j}}$ are $\mathcal{C N}\left(0, \sigma_{i n_{j}}^{2}\right)$ and $\mathcal{C N}\left(0, N_{0}\right)$ distributed, respectively. At the destination, the received signal from both phases are combined using the maximum ratio combining (MRC), then the instantaneous SNR at the MRC output is

$$
\gamma_{j}=\left(P_{j j}\left|h_{j n_{j}}\right|^{2}+\tilde{P}_{i j}\left|h_{i n_{j}}\right|^{2}\right) / N_{0},
$$

where $x_{j}$ is assumed to have unit energy. In case of BPSK signaling, the conditional BER based on $\gamma_{j}$ is $\operatorname{BER}^{h}\left(\gamma_{j}\right)=$ $(1 / \pi) \int_{0}^{\pi / 2} \exp \left(-\gamma_{j} / \sin ^{2} \theta\right) d(\theta)$, [10] where $\gamma_{j}$ is the instantaneous SNR obtained in (4). Therefore, the chance of incorrectly decoding at the relay is $\operatorname{BER}^{h}\left(P_{j j}\left|h_{j i}\right|^{2} / N_{0}\right)$, while the chance of correctly decoding at the relay is $1-\operatorname{BER}^{h}\left(P_{j j}\left|h_{j i}\right|^{2} / N_{0}\right)$. By taking into account these two chances and averaging the conditional BER over all Rayleigh fading channels, the average BER in case of BPSK signaling is [10]

$$
\begin{aligned}
\mathrm{BER}_{j}= & F\left(1+P_{j j} \sigma_{j n_{j}}^{2} /\left(N_{0} \sin ^{2} \theta\right)\right) \cdot F\left(1+P_{j j} \sigma_{j i}^{2} /\left(N_{0} \sin ^{2} \theta\right)\right) \\
& +F\left(\left(1+P_{j j} \sigma_{j n_{j}}^{2} /\left(N_{0} \sin ^{2} \theta\right)\right)\left(1+P_{i j} \sigma_{i n_{j}}^{2} /\left(N_{0} \sin ^{2} \theta\right)\right)\right) \\
& \times\left[1-F\left(1+P_{j j} \sigma_{j i}^{2} /\left(N_{0} \sin ^{2} \theta\right)\right)\right]
\end{aligned}
$$

where $F(x(\theta))=\frac{1}{\pi} \int_{0}^{\pi / 2}[x(\theta)]^{-1} d \theta$. The first term on the right hand side of (5) corresponds to the case of incorrectly decoding at the relay, and the second term corresponds to the case of correctly decoding at the relay. By assuming that all channel links are available, i.e., $\sigma_{j n_{j}}^{2} \neq 0$ and $\sigma_{j i}^{2} \neq 0$, the BER upper bound of the closed form BER expression in (5) can be obtained by removing the negative term and all one's in (5). The resulting BER upper bound is given by [10]

$$
\operatorname{BER}_{j} \leq N_{0}^{2}\left(\left(A^{2} P_{i j} \sigma_{i n_{j}}^{2}+B P_{j j} \sigma_{j i}^{2}\right) /\left(P_{j j}^{2} P_{i j} \sigma_{j n_{j}}^{2} \sigma_{j i}^{2} \sigma_{i n_{j}}^{2}\right)\right),
$$

where $A=F\left(\sin ^{-2} \theta\right)=0.25$ and $B=F\left(\sin ^{-4} \theta\right)=0.1875$. Note that when the sensors transmit non-cooperatively, the BER upper bound [10] is given by

$$
\mathrm{BER}_{j} \leq N_{0} /\left(4 P_{i i} \sigma_{j n_{j}}^{2}\right) .
$$

\section{Lifetime Maximization By Cooperative Sensor}

We first formulate the lifetime maximization problem, then provide analytical solution for a two-sensor system network, and finally develop a fast suboptimal algorithm for multiple-sensor scenario.

For subsequent evaluation, we define a power allocation matrix $\mathbf{P}$ as an $N \times N$ matrix with the following properties:

${ }^{1}$ Practically, this can be done at the relay by applying a simple SNR threshold on the received data. Although, it can lead to some error propagation, but for practical ranges of operating SNR, the event of error propagation are negligible.
1) Each element $P_{i j} \geq 0, \forall i, j=1,2, \cdots, N$.

2) $P_{j j}$ represents the power that sensor $j$ uses to transmit information to the next node $n_{j}$ and the relays.

3) $P_{i j}$ represents the power that sensor $i$ helps forward information of sensor $j$ to $n_{j}$.

We assume that all sensors have the information to be transmitted, i.e. $P_{j j}>0$. To maintain bandwidth efficiency, we assume that each sensor can be helped only once. Accordingly, each column of $\mathbf{P}$ contains at most two non-zero elements, i.e., $\sum_{i=1}^{N} \operatorname{sgn}\left(P_{i j}\right) \leq$ 2 , where $\operatorname{sgn}(x)$ returns 1 if $x>0$, and 0 if $x=0$.

\section{A. Problem Formulation}

We use in this subsection the definition of network lifetime in [1] which is the duration from the deployment of the network to the time that the battery of the first node is fully drained. Other definitions of network lifetime can be applied in a similar way. Denote $P_{s}$ as a processing power at the source and $P_{r}$ as a processing power at the relay. Also, let $\lambda_{l i}$ be the transmission rate from node $l$ to node $i$. We consider the scenario where all sensors transmit to one common destination (e.g., processing center or base station). The overall transmitted power of the relay (sensor $i$ ) is $P_{i i} \sum_{l=1}^{N} \lambda_{l i}+$ $\sum_{j=1, j \neq i}^{N} P_{i j}\left(\sum_{l=1}^{N} \lambda_{l j}\right)$, and the overall processing power of sensor $i$ is $\lambda_{i i} P_{s}+\sum_{j=1, j \neq i}^{N} P_{r} \operatorname{sgn}\left(P_{i j}\right)\left(\sum_{l=1}^{N} \lambda_{l j}\right)$ where $\operatorname{sgn}(\cdot)$ is the sign function. Hence, the lifetime of the cooperating sensor $i$ is

$$
T_{i}=E_{i} / \mathcal{P}_{i}
$$

where $\mathcal{P}_{i} \triangleq \lambda_{i i} P_{s}+P_{i i} \sum_{l=1}^{N} \lambda_{l i}+\sum_{j=1, j \neq i}^{N}\left(P_{r} \operatorname{sgn}\left(P_{i j}\right)+\right.$ $\left.P_{i j}\right)\left(\sum_{l=1}^{N} \lambda_{l j}\right), E_{i}$ is the initial battery energy of sensor $i$. Accordingly, the network lifetime is given by

$$
T_{\text {net }}(\mathbf{P})=\min _{i} T_{i}
$$

Whence, the maximum lifetime problem can be formulated as

$$
\begin{gathered}
\hat{T}_{\text {net }}=\max \min _{i} T_{i}=\max _{\mathbf{P}} T_{n e t}(\mathbf{P}) \\
\text { s.t. }\left\{\begin{array}{l}
\text { Performance: } \mathrm{BER}_{i} \leq \varepsilon_{i}, \forall i ; \\
\text { Cooperation: } \sum_{i=1}^{N} \operatorname{sgn}\left(P_{i j}\right) \leq 2, \forall j ; \\
\text { Power: } 0<P_{i i} \leq P_{\max }, \forall i ; \\
\text { Power: } 0 \leq P_{i j} \leq P_{\max }, \forall j \neq i,
\end{array}\right.
\end{gathered}
$$

where the first constraint is to satisfy the BER performance requirement $\left(\varepsilon_{i}\right)$, the second constraint is to ensure that each sensor can be helped only once, the third constraint is to guarantee that each sensor has information to transmit and the transmitted power is no greater than $P_{\max }$, and the fourth constraint is to ensure that all the allocated powers are non-negative and no greater than $P_{\text {max }}$. We assume, without loss of generality, that the non-cooperative transmit power of each sensor is feasible, i.e. $P_{i i}$ is always less than $P_{\max }$.

\section{B. Analytical Solution for a Two-Sensor WSN}

To better understand the formulated problem, we provide in this section an analytical solution for a two-sensor cooperative WSN. Both cooperative sensors transmit information directly to the same destination $d$. Under this scenario, there are three possible transmission strategies which will be explained in the sequel. The optimum power allocation is the one that results in the maximum network lifetime over all possible transmission strategies.

1) Non-cooperative transmission among sensors: Based on (7), the optimum power allocation for non-cooperative case is

$$
P_{j j}=N_{0} /\left(4 \varepsilon_{j} \sigma_{j d}^{2}\right)
$$


for $j=1,2$, and $P_{i j}=0$ for $i \neq j$. Substituting (11) into (9), the network lifetime for non-cooperative transmission is

$$
\hat{T}_{\text {non-coop }}=\min \left[E_{1} /\left(\lambda_{11}\left(P_{s}+P_{11}\right)\right), E_{2} /\left(\lambda_{22}\left(P_{s}+P_{22}\right)\right)\right] \text {. }
$$

2) Cooperative transmission when one sensor helps the other sensor: Without loss of generality, we will provide the solution for a case that sensor $i$ helps relay information of sensor $j$. The lifetimes of sensor $i$ and sensor $j$ are given by

$$
T_{i}=E_{i} /\left(\lambda_{i i}\left(P_{s}+P_{i i}\right)+\lambda_{j j}\left(P_{r}+P_{i j}\right)\right),
$$

and

$$
T_{j}=E_{j} /\left(\lambda_{j j}\left(P_{s}+P_{j j}\right)\right) .
$$

In order for sensor $i$ to satisfy the BER requirement $\varepsilon_{i}$, the optimum transmitted power of sensor $i\left(P_{i i}\right)$ is given by (11) by replacing $j$ with $i$. For sensor $j$, however, the optimum powers $P_{j j}$ and $P_{i j}$ are such that $\varepsilon_{j}=\left(N_{0}^{2} A^{2} P_{i j} \sigma_{i d}^{2}+\right.$ $\left.N_{0}^{2} B P_{j j} \sigma_{j i}^{2}\right) /\left(P_{j j}^{2} P_{i j} \sigma_{j d}^{2} \sigma_{j i}^{2} \sigma_{i i}^{2}\right)$, and we can express $P_{i j}$ in terms of $P_{j j}$ as

$$
P_{i j}=P_{j j} /\left(C_{i j} P_{j j}^{2}-D_{i j}\right) \triangleq f\left(P_{j j}\right),
$$

where $C_{i j}=\varepsilon_{2} \sigma_{i d}^{2} \sigma_{j d}^{2} /\left(B N_{0}^{2}\right)$ and $D_{i j}=A^{2} \sigma_{i d}^{2} /\left(B \sigma_{j i}^{2}\right)$. By substituting $P_{i j}$ from (15) and $P_{i i}$ from (11) into (13), the optimization problem in (10) is simplified to

$$
\begin{aligned}
\hat{T}_{i-h e l p s-j}=\max _{P_{j j}}[ & \min \left(\frac{E_{i}}{\lambda_{i i}\left(P_{s}+\frac{N_{0}}{4 \varepsilon_{i} \sigma_{i d}^{2}}\right)+\lambda_{j j}\left(P_{r}+f\left(P_{j j}\right)\right)},\right. \\
& \left.\left.\frac{E_{j}}{\lambda_{j j}\left(P_{s}+P_{j j}\right)}\right)\right]
\end{aligned}
$$

such that $P_{j j} \leq P_{\max }$ and $P_{i j}=f\left(P_{j j}\right) \leq P_{\max }$.

For unconstraint optimization of (16), it can be shown that the optimal power $P_{j j}\left(\hat{P}_{j j}\right)$ is the one that results in $T_{i}=T_{j}$. Hence $\hat{P}_{j j}$ is the solution to

$$
C_{i j} E_{i} \lambda_{j j} P_{j j}^{3}+K C_{i j} P_{j j}^{2}-\left(D_{i j} \lambda_{j j} E_{i}+\lambda_{j j} E_{j}\right) P_{j j}-K D_{i j}=0,
$$

in which $K=E_{i} \lambda_{j j} P_{s}-E_{j} \lambda_{i i} P_{s}-\lambda_{j j} E_{j} P_{r}+$ $\left(\lambda_{i i} N_{0} E_{j}\right) /\left(4 \varepsilon_{i} \sigma_{i d}^{2}\right)$. Accordingly, we can find that $\tilde{P}_{i j}=f\left(\tilde{P}_{j j}\right)$ from the relation in (15). Therefore, the unconstraint maximum network lifetime in case of sensor $i$ helps sensor $j$ is given by

$$
\tilde{T}_{i-h e l p s-j}=E_{j} /\left(\lambda_{j j}\left(P_{s}+\tilde{P}_{j j}\right)\right),
$$

If the constraint on transmitted power is such that $\tilde{P}_{i j} \leq P_{\max }$, we can use (18) as the optimum network lifetime for this scenario. However, if $\tilde{P}_{i j}>P_{\max }$, we choose $\tilde{P}_{i j}=P_{\max }$. In this way, we can find $\tilde{P}_{j j}$ through the relation of $\tilde{P}_{j j}$ and $\tilde{P}_{i j}$ in (6):

$$
\tilde{P}_{j j}=\left(-Q_{1}+\sqrt{Q_{1}^{2}+Q_{2} Q_{3} P_{\max }^{2}}\right) /\left(Q_{2} P_{\max }\right),
$$

where $Q_{1}=B \sigma_{j i}^{2} N_{o}^{2}, Q_{2}=2 \varepsilon_{j} \sigma_{i d}^{2} \sigma_{j i}^{2} \sigma_{j d}^{2}$, and $Q_{3}=2 A^{2} \sigma_{i d}^{2} N_{o}^{2}$. In this scenario, the lifetime of sensor $i$ and sensor $j$ are

$$
\begin{aligned}
& \tilde{T}_{i}=E_{i} /\left(\lambda_{i i}\left(P_{s}+P_{i i}\right)+\lambda_{j j}\left(P_{r}+P_{\max }\right)\right), \\
& \tilde{T}_{j}=E_{j} /\left(\lambda_{j j}\left(P_{s}+\tilde{P}_{j j}\right)\right),
\end{aligned}
$$

respectively, and the optimum lifetime when $\tilde{P}_{i j}>P_{\max }$ is the minimum among $\tilde{T}_{i}$ and $\tilde{T}_{j}$. Therefore, the optimum network lifetime when sensor $i$ helps sensor $j$ is

$$
\hat{T}_{i-\text { helps-j }}= \begin{cases}E_{j} /\left(\lambda_{j j}\left(P_{s}+\tilde{P}_{j j}\right)\right), & \tilde{P}_{i j} \leq P_{\max } \\ \min \left\{\tilde{T}_{i}, \tilde{T}_{j}\right\}, & \tilde{P}_{i j}>P_{\max }\end{cases}
$$

3) Cooperative transmission when both sensors help each other: In this scenario, the power that sensor $i$ helps sensor $j$
$\left(P_{i j}\right)$ can be obtained in the same way as in (15), and the optimum network lifetime can be obtained by finding $\tilde{P}_{i i}$ and $\tilde{P}_{j j}$ that maximizes $T_{i}$ under the condition: $T_{i}=T_{j}$. Hence

$$
\begin{aligned}
\tilde{T}_{\text {both-help }} & =\frac{E_{i}}{\lambda_{i i}\left(P_{s}+\tilde{P}_{i i}\right)+\lambda_{j j}\left(P_{r}+\left(\tilde{P}_{j j} /\left(C_{i j} \tilde{P}_{j j}^{2}-D_{i j}\right)\right)\right.}, \\
& =\frac{E_{j}}{\lambda_{j j}\left(P_{s}+\tilde{P}_{j j}\right)+\lambda_{i i}\left(P_{r}+\left(\tilde{P}_{i i} /\left(C_{j i} \tilde{P}_{i i}^{2}-D_{j i}\right)\right)\right.},
\end{aligned}
$$

where $\tilde{P}_{i i}$ and $\tilde{P}_{j j}$ are the solutions to the optimization problem:

$$
\begin{aligned}
& \tilde{T}=\max _{P_{i i}, P_{j j}} \frac{E_{i}}{\lambda_{i d}\left(P_{s}+P_{i i}\right)+\lambda_{j i}\left(P_{r}+P_{j j} /\left(C_{i j} P_{j j}^{2}-D_{i j}\right)\right)} \\
& \text { s.t. }\left\{\begin{array}{l}
\frac{\left[\left(\lambda_{i i} P_{s}+\lambda_{j j} P_{r}+\lambda_{i i} P_{i i}\right)\left(C_{i j} P_{j j}^{2}-D_{i j}\right)+\lambda_{j j} P_{j j}\right]\left(C_{j i} P_{i i}^{2}-D_{j i}\right)}{\left[\left(\lambda_{j j} P_{s}+\lambda_{i i} P_{r}+\lambda_{j j} P_{j j}\right)\left(C_{j i} P_{i i}^{2}-D_{j i}\right)+\lambda_{i i} P_{i i}\right]\left(C_{i j} P_{j j}^{2}-D_{i j}\right)}=\frac{E_{i}}{E_{j}} ; \\
P_{j j}>\sqrt{D_{i j} / C_{i j}}, \forall j \neq i .
\end{array}\right.
\end{aligned}
$$

In (24), the first constraint is to ensure that $T_{i}=T_{j}$, and the second constraint is to guarantee that $P_{i j}=P_{j j} /\left(C_{i j} P_{j j}^{2}-\right.$ $\left.D_{i j}\right)>0$. Note that (24) is a convex optimization problem, hence, the solution can be found by any available optimization algorithm. If $\max \left\{\tilde{P}_{i j}, \tilde{P}_{j i}\right\} \leq P_{\text {max }}$, then the solution to (23) is the optimal network lifetime. Otherwise, the optimal network lifetime can be solved based on the boundary conditions as in Section III-B.2.

Finally the optimal network lifetime for the two-sensor WSN can be obtained from (12), (18), and (23) by

$$
\hat{T}_{\text {net }}=\max \left\{\hat{T}_{\text {non-coop }}, \hat{T}_{1-\text { helps-2 }}, \hat{T}_{2-\text { helps-1 }}, \hat{T}_{\text {both-help }}\right\} .
$$

\section{Suboptimal Algorithm for Multiple Sensor WSN}

As the number of sensors increases, although the optimal solution can be obtained through full search, it is computationally expensive. We propose, in this subsection, a suboptimal greedy algorithm to determine the network lifetime.

We first consider the solution to problem for the multiple-sensor WSN. In this case, the optimization problem can be written as

$$
\begin{gathered}
\hat{T}_{i-h e l p s-j}=\max _{P_{j j}}\left[\operatorname { m i n } \left(\frac{E_{i}}{K_{i}+\left(P_{r}+f\left(P_{j j}\right)\right) \sum_{l=1}^{N} \lambda_{l j}},\right.\right. \\
\left.\left.\frac{E_{j}}{K_{j}+P_{j j} \Sigma_{l=1}^{N} \lambda_{l j}}\right)\right]
\end{gathered}
$$

where $K_{i}=\lambda_{i i} P_{s}+P_{i i} \Sigma_{l=1}^{N} \lambda_{l i}+\Sigma_{k=1, k \neq i, j}^{N}\left(P_{r} \operatorname{sgn}\left(P_{i k}\right)+\right.$ $\left.P_{i k}\right)\left(\Sigma_{l=1}^{N} \lambda_{l k}\right)$, and $K_{j}=\lambda_{j j} P_{s}+\Sigma_{k=1, k \neq j}^{N}\left(P_{r} \operatorname{sgn}\left(P_{j k}\right)+\right.$ $\left.P_{j k}\right)\left(\sum_{l=1}^{N} \lambda_{l k}\right)$, in which $K_{i}$ and $K_{j}$ are constants that includes the processing power, the power for transmitting its own information and forwarding information of others, and the transmitted powers for cooperative transmission. Similar to (18), we can find that the unconstraint maximum network lifetime when sensor $i$ helps sensor $j$ is given by

$$
\tilde{T}_{i-h e l p s-j}=E_{j} /\left(K_{j}+\tilde{P}_{j j} \Sigma_{l=1}^{N} \lambda_{l j}\right),
$$

where $\tilde{P}_{j j}$ is the solution to $C_{i j} E_{i} \Sigma_{l=1}^{N} \lambda_{l j} P_{j j}^{3}+G C_{i j} P_{j j}^{2}-$ $\left(D_{i j} E_{i}+E_{j}\right)\left(\sum_{l=1}^{N} \lambda_{l j}\right) P_{j j}-G D_{i j}=0$, in which $G=E_{i} K_{j}-$ $E_{j} K_{i}-E_{j} P_{r} \Sigma_{l=1}^{N} \lambda_{l j}$. Using (27), the optimum network lifetime for the multiple-sensor WSN can be obtained in a similar way as (19)-(22), and the solution is used to determine lifetime of each sensor in the proposed suboptimal algorithm which has the following descriptions.

Initially, the power allocation matrix $\mathbf{P}$ is assigned as a diagonal matrix with its diagonal component $P_{j j}$ follows (11), i.e., the initial scheme is the non-cooperative transmission scheme. The corresponding lifetime of sensor $j$ is $T_{j}=E_{j} /\left(\lambda_{j j} P_{s}+\right.$ $\left.P_{j j} \Sigma_{l=1}^{N} \lambda_{l j}\right)$. Construct a helped list which is a list of all possible 
TABLE I: Suboptimal algorithm for maximum network lifetime of WSN with multiple cooperative sensors

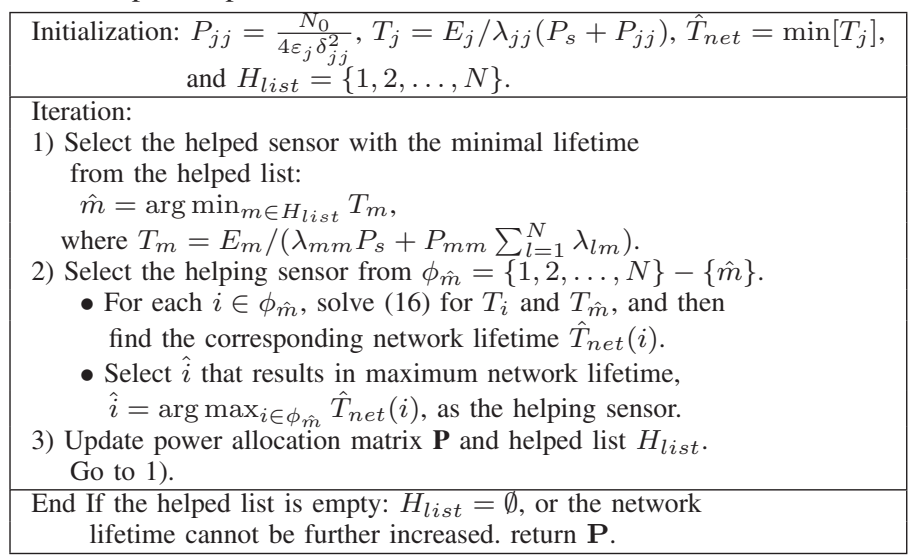

sensors to be helped: $H_{\text {list }}=\{1,2, \ldots, N\}$. First, the algorithm finds the helped sensor from the helped list by choosing the sensor who has minimum lifetime, i.e., the helped sensor $\hat{m}$ is given by

$$
\hat{m}=\arg \min _{m \in H_{\text {list }}} T_{m} .
$$

Second, the algorithm finds a sensor to help sensor $\hat{m}$ by choosing among all sensors $i, i=1,2, \ldots, N$ and $i \neq \hat{m}$. For each possible helping sensor $i$, the algorithm uses (16) to find power allocation for the helping sensor $i$ and the helped sensor $\hat{m}$. After that, the algorithm determines lifetime $\hat{T}_{n e t}(i)$ after sensor $i$ helps sensor $\hat{m}$ by choosing the minimum lifetime among lifetimes of all sensors in the networks, i.e., $\hat{T}_{n e t}(i)=\min _{i}\left[T_{1}, T_{2}, \cdots, T_{\hat{m}}\right]$. The obtained $\hat{T}_{n e t}(i), i=1,2, \cdots, N-1$, from all possible helping sensors are compared, and the algorithm selects sensor $\hat{i}$ that leads to maximum network lifetime to help sensor $\hat{m}$. Next, the algorithm updates the power allocation matrix $\mathbf{P}$ and updates the helped list by removing sensor $\hat{m}$ from the helped list. Then, the algorithm goes back to determine the helped sensor again from the updated helped list. This iteration is continued until all sensors have been helped, i.e., the helped list is empty, or the network lifetime cannot be further increased. The resulting $\mathbf{P}$ is the power allocation matrix which gives answer to the questions which sensor should help which sensor and how much power to cooperate. The detailed algorithm is shown in Table I.

Although the proposed algorithm is suboptimal, we will show in Section V that the algorithm significantly improves the network lifetime. Moreover, the proposed algorithm can be implemented at a center node to manage power allocation and cooperation strategy of all sensors. The cooperative sensors follow the executed algorithm, and hence there is no additional computation burden at the sensors. In addition, the proposed algorithm can be directly extended to a larger WSN; the complexity of the algorithm is only linearly increased with the number of sensors.

\section{Cooperative Relay Deployment}

In this section, we study the network lifetime by the deployment of cooperative relays in an energy depleting WSN. The lifetime maximization problem is formulated, and an algorithm to solve the problem is explained.

\section{A. Problem Formulation}

We consider a WSN with $N$ randomly-located sensors, $K$ relays, and a destination. Since we assume that there is no cooperation among the sensors, the power allocation matrix $\mathbf{P}$ as defined in Section III is an $N \times N$ diagonal matrix whose each diagonal element $P_{j j}>0$ for all $j$. The lifetime of noncooperative sensor $j$ can be expressed as in (14). We also define $\hat{\mathbf{P}}$ as a $K \times N$ relay power allocation matrix whose $(i, j)^{t h}$ element, $\hat{P}_{i j}$, represents the power that the relay $R_{i}$ helps the sensor $j$. To simplify the analysis, we assume that each sensor is helped by only one relay. This implies that each column of the matrix $\hat{\mathbf{P}}$ contains at most one non-zero element. Denote $E_{R_{i}}$ as the initial energy of relay $R_{i}$, the lifetime of relay $R_{i}$ is

$$
T_{R_{i}}=E_{R_{i}} /\left(\sum_{j=1}^{N}\left(P_{r} \operatorname{sgn}\left(\hat{P}_{i j}\right)+\hat{P}_{i j}\right)\left(\sum_{l=1}^{N} \lambda_{l j}\right)\right) .
$$

Let $x_{j}$ and $y_{j}$ be the locations of sensor $j$ on the $\mathrm{x}$-axis and the y-axis, respectively, then the location of sensor $j$ is represented by a vector $\overline{\mathbf{D}}_{j}=\left[\begin{array}{ll}x_{j} & y_{j}\end{array}\right]^{T}$. We assume that the next routing node of node $j$ is located at $\bar{D}_{n_{j}}=\left[\begin{array}{ll}x_{n_{j}} & y_{n_{j}}\end{array}\right]^{T}$. Therefore, the channel variance between sensor $j$ and $n_{j}$ is $\sigma_{j n_{j}}^{2}=k\left\|\overline{\mathbf{D}}_{j}-\overline{\mathbf{D}}_{n_{j}}\right\|^{-\alpha}$ where $\|\cdot\|$ denotes the Frobenius norm [14]. Similarly, the locations of the relays are specified by a $2 \times K$ matrix $\mathbf{D}_{R}=\left[\begin{array}{llll}\overline{\mathbf{D}}_{R_{1}} & \overline{\mathbf{D}}_{R_{2}} & \cdots & \overline{\mathbf{D}}_{R_{K}}\end{array}\right]$ in which $\bar{D}_{R_{i}}=\left[x_{R_{i}} y_{R_{i}}\right]^{T}$ and the $i^{t h}$ column indicates the location of relay $R_{i}$. Accordingly, the channel variance between relay $R_{i}$ and node $n_{j}$ is $\sigma_{R_{i}, n_{j}}^{2}=k\left\|\overline{\mathbf{D}}_{R_{i}}-\overline{\mathbf{D}}_{n_{j}}\right\|^{-\alpha}$, and the channel variance between sensor $j$ and relay $R_{i}$ is $\sigma_{j, R_{i}}^{2}=k\left\|\overline{\mathbf{D}}_{j}-\overline{\mathbf{D}}_{R_{i}}\right\|^{-\alpha}$. If sensor $j$ is helped by relay $R_{i}$, then the BER performance of sensor $j$ is given by (5) with $P_{i j}$ and $\sigma_{j i}^{2}$ replaced by $\hat{P}_{i j}$ and $\sigma_{j, R_{i}}^{2}$, respectively.

Given $\mathbf{D}_{R}, \mathbf{P}$, and $\hat{\mathbf{P}}$; the network lifetime can be expressed as $T_{n e t}\left(\mathbf{D}_{R}, \mathbf{P}, \hat{\mathbf{P}}\right)=\min _{i, j}\left\{T_{j}, T_{R_{i}}\right\}$., and the maximum lifetime optimization problem can be formulated as

$$
\begin{gathered}
\hat{T}=\max _{\mathbf{D}_{R}, \mathbf{P}, \hat{\mathbf{P}}} T_{n e t}\left(\mathbf{D}_{R}, \mathbf{P}, \hat{\mathbf{P}}\right) \\
\text { s.t. }\left\{\begin{array}{l}
\text { Performance: } \mathrm{BER}_{j} \leq \varepsilon_{j}, \forall j ; \\
\text { Cooperation: } \sum_{i=1}^{K} \operatorname{sgn}\left(\hat{P}_{i j}\right) \leq 1, \forall j ; \\
\text { Power: } 0<P_{i i} \leq P_{\max }, P_{i j}=0 \forall i, j \neq i ; \\
\text { Power: } 0 \leq \hat{P}_{i j} \leq P_{\text {max }}, \forall i, j .
\end{array}\right.
\end{gathered}
$$

In (30), the first constraint is to satisfy the BER requirement. The second constraint is to ensure that each sensor is helped by at most one relay. The third constraint is to guarantee that all sensors transmit their information with powers no greater than $P_{\max }$ and there is no cooperation among sensors. The forth constraint is to ensure that the powers that the relays help the sensors are nonnegative and no greater than $P_{\max }$. It turns out that the formulated problem in (30) is NP hard. Since it is computationally expensive to obtain the optimal solution to (30), we propose in the next subsection a fast suboptimal algorithm.

\section{B. Proposed Algorithm}

In what follows, we describe the algorithm to determine the network lifetime in each step for fixed locations and fixed number of the cooperative relays. Then an algorithm to find the relay locations is provided.

To obtain the network lifetime when the number of relays and their locations are given, we use greedy suboptimal algorithm as follows. Initially, all sensors are sorted according to their noncooperative lifetimes (14) in ascending order, and the sensors are listed in a helped list $H_{\text {list }}$. In each iteration, first, select the first sensor in the helped list as the one to be helped. Second, 
TABLE II: Suboptimal algorithm to determine network lifetime when relay locations are fixed

\begin{tabular}{|l}
\hline Initialization: $P_{j j}=\frac{N_{0}}{4 \varepsilon_{j} \sigma_{j j}^{2}}, T_{j}=\frac{E_{j}}{\lambda_{j j}\left(P_{s}+P_{j j}\right)}, \hat{T}_{n e t}=\min \left[T_{j}\right]$, \\
Sort $N$ sensors by their lifetimes in \\
ascending order and list in $H_{l i s t}$.
\end{tabular}

TABLE III: Algorithm to determine relay locations

Initialization: $q=0, \Phi_{D}=\left\{\mathbf{D}_{1}, \mathbf{D}_{2}, \ldots, \mathbf{D}_{L}\right\}$
Iteration:
1) Increase number of relays: $\mathbf{q}=\mathrm{q}+1$
2) For each location $\mathbf{D}_{l} \in \Phi_{D}$. Set $\mathbf{D}_{R_{q}}=\mathbf{D}_{l}$.
Find $\hat{T}_{n e t}(l), \mathbf{P}(l)$ and $\hat{\mathbf{P}}(l)$ using the algorithm in Table II
3) Find the relay location $R_{q}: \mathbf{D}_{R_{q}}=\mathbf{D}_{\hat{l}}$, where
$\quad \hat{l}=\arg \max _{l} \hat{T}_{n e t}(l)$
4) Update $\mathbf{P}, \hat{\mathbf{P}}$, and $\hat{T}_{n e t}$
Remove the location $\mathbf{D}_{\hat{l}}$ from the set $\Phi_{D}$. Go to 1).
End: If the network lifetime cannot be further increased,
$\quad$ or $q=K_{\max }$. Return $\mathbf{P}, \hat{\mathbf{P}}, \hat{T}_{n e t}$.

determine the network lifetime $T_{\text {net }}(i)$ after all of the relay $R_{i}$ 's $(i=1,2, \ldots, K)$ help the selected sensor, and then choose the relay $R_{\hat{i}}$ that results in maximum network lifetime to help the selected sensor. Next, update the power allocation matrices $\mathbf{P}$ and $\hat{\mathbf{P}}$, remove the selected sensor from the helped list, and go back to the first step. The iteration continues until all sensors have been helped and the helped list is empty or until the network lifetime cannot be further improved.

The proposed algorithm for finding the relay locations are as follows. We denote $K_{\max }$ as the maximum number of relays, $L$ as the total number of possible relay locations, and $\left\{\mathbf{D}_{1}, \mathbf{D}_{2}, \ldots, \mathbf{D}_{L}\right\}$ as the set of all possible relay locations. Initially, the number of relays is set to zero, and the set of all possible relay locations is $\Phi_{D}=\left\{\mathbf{D}_{1}, \mathbf{D}_{2}, \ldots, \mathbf{D}_{L}\right\}$. In each iteration, the number of relay is increased by one. For each fixed location $\mathbf{D}_{l} \in \Phi_{D}$, the algorithm in Table II is used to obtain $\hat{T}_{n e t}(l)$ and the corresponding $\mathbf{P}(l)$ and $\hat{\mathbf{P}}(l)$. Next, the location $\hat{l}$ that results in maximum network lifetime is selected as relay location. Then, the network lifetime is updated, and the location $D_{\hat{l}}$ is removed from the set $\Phi_{D}$. Finally, the algorithm goes back to the first step. The algorithm stops if the network lifetime can not be improved or the number of relays reaches $K_{\max }$. The detailed algorithm is presented in Table III.

\section{Simulation Results And Discussions}

In all simulations, the propagation loss factor is $\alpha=2$, and the BER requirement of each sensor is $\varepsilon_{j}=10^{-3}$. The processing power of each sensor $\left(P_{s}\right)$ is set at $25 \%$ of the average transmitted power of the sensor. The processing power of each relay $\left(P_{r}\right)$ is set at $50 \%$ of $P_{s}$. All sensor have equal initial battery energies of $E_{j}=10^{5}$. Unless stated otherwise, the sensors are randomly distributed based on uniform distribution over the considered area,

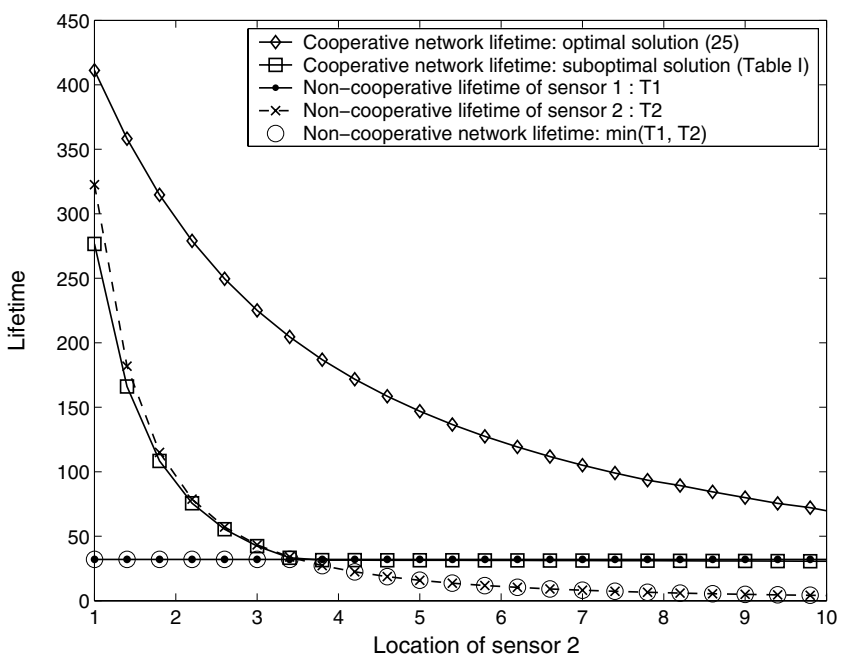

Fig. 1: Lifetimes of sensors in the two-sensor WSN.

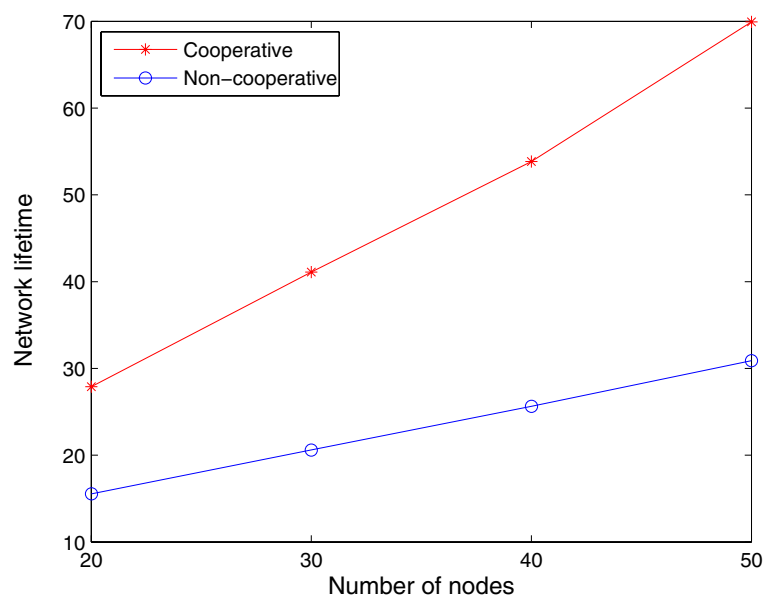

Fig. 2: Network lifetime of a WSN with randomly located sensors.

and the base station is located in the center. Each sensor send information to the base station via a route that is determined using Dijkstra's shortest path algorithm.

In Figure 1, we consider a two-sensor WSN, where sensor 1 is located at coordinate $(0,3.5)$. The location of sensor 2 varies from $(0,1)$ to $(0,9)$. We can see that the network lifetime of noncooperative scheme is determined by the lifetime of the sensor who is located farther from the destination. Under cooperative transmission, the network lifetime is significantly increased, especially when sensor 2 is located close to the destination. This is because sensor 2 requires small transmitted power to reach the destination; after sensor 2 helps sensor 1, the transmitted power of sensor 2 slightly increases, while the transmitted power of sensor 1 greatly reduces due to the cooperation diversity. With the proposed suboptimal approach (Table II), the network lifetime is improved to almost the same as the lifetime of the sensor who is closer to the destination. By using the optimal power allocation obtained in Section III-B, the network lifetime can be further increased since both sensors take advantage of the cooperation diversity while using smaller amount of their transmitted powers.

Figure 2 depicts the network lifetime of WSN with multiple cooperative sensors. The number of sensors varies from 20 to 50 , and the monitoring area is of size $100 \mathrm{~m} \times 100 \mathrm{~m}$. Clearly, the network lifetime of cooperative scheme is larger than that of 


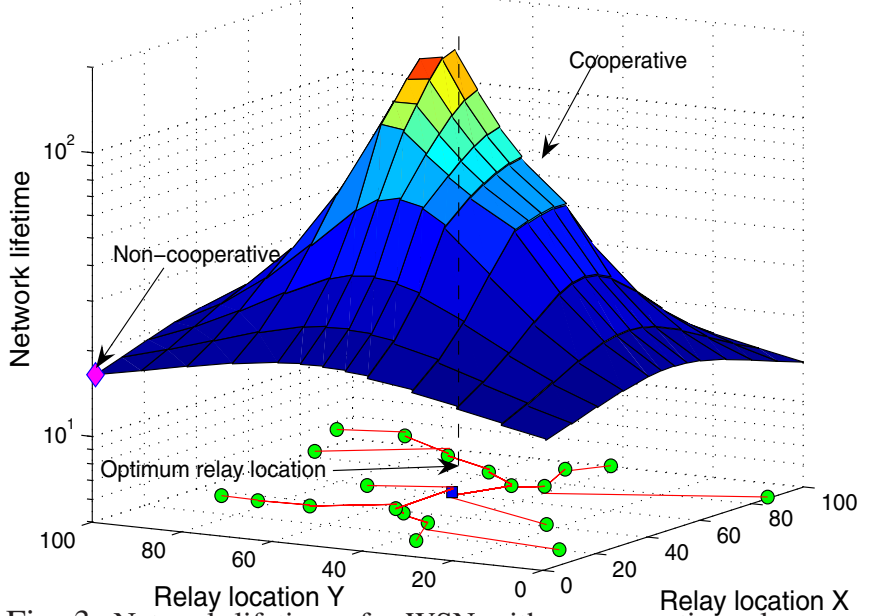

Fig. 3: Network lifetime of a WSN with a cooperative relay.

non-cooperative scheme for any number of sensors. For example, for a WSN with 50 sensors, the cooperative scheme improves the network lifetime by 2.3 times that of non-cooperative scheme. Note that the network lifetime of both schemes decreases with the number of sensors since we assume that all sensors have information to transmit; the more the sensors, the more the packets each node needs to send on its route.

Figure 3 shows the network lifetime of a WSN with a cooperative relay deployment. We consider a monitoring area of $100 \mathrm{~m} \times 100 \mathrm{~m}$ that contains 20 sensors (each represented by a circle node). Each of the sensors transmits information through a predetermined route to the base station that is located at the middle of the area (represented by a rectangular node). The cooperative relay is equipped with initial energy of $E_{R_{i}}=10^{6}$. In the figure, each point on the $\mathrm{x}$-axis and the $\mathrm{y}$-axis represents the relay location at coordinate $(x, y)$, and the $\mathrm{z}$-axis is the network lifetime according to each relay location. We can see that the network lifetime of the cooperative WSN gradually improves as location of the relay is closer to the center of the area. At the optimum relay location, the cooperative WSN increases network lifetime by 9 times that of the non-cooperative WSN. This is because the sensor that is nearest to the destination tends to die first, and its lifetime can be greatly improved by the relay that is located close to the center.

Figure 4, shows the network lifetime as a function of the number of cooperative relays. We consider a WSN with 20 sensors in a $100 \mathrm{~m} \times 100 \mathrm{~m}$ monitoring area. The initial energy of each relay is $10^{5}$. By randomly deploying a cooperative relay, the cooperative WSN achieves about 2.4 times longer network lifetime than the non-cooperative WSN. If the relay is placed at the optimum location, the network lifetime is further improved to 3 times that of the non-cooperative WSN.

\section{CONCLUSIONS}

In this paper, we propose the cooperative sensor/relay deployment in wireless sensor network (WSN). With cooperative WSN, energy consumption is evenly distributed among cooperative sensors as well as cooperation diversity is exploited such that the network lifetime is improved. First, decode-andforward cooperation protocol is employed among sensors. We determine which sensors should cooperate and how much power

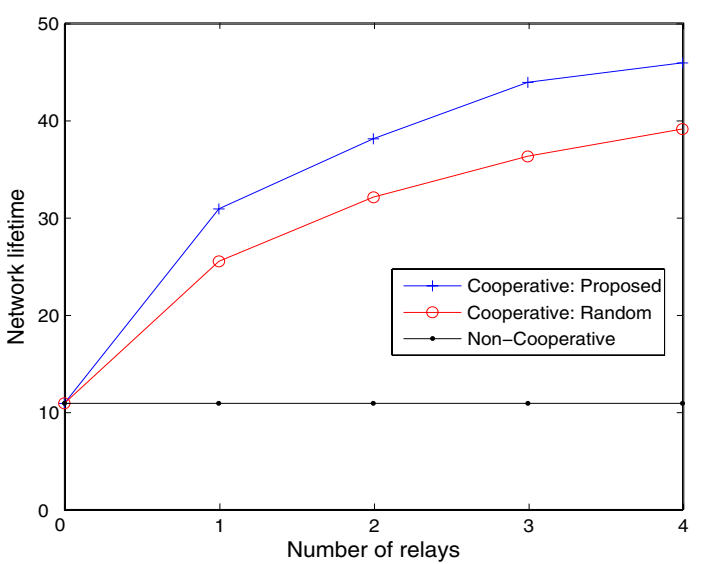

Fig. 4: Network lifetime of the proposed cooperative WSN where different number of cooperative relays are deployed.

to allocate for cooperation. An analytical solution for a two-sensor WSN is provided. A suboptimal algorithm for a multiple sensor WSN is developed to reduce the complexity of the formulated problem. Simulation results show that the network lifetime of the multiple sensor WSN with random locations increases to 2.3 times compared with that of the non-cooperative WSN. In addition, we consider improving the network lifetime by adding cooperative relays into an energy depleting WSN. A suboptimal algorithm is developed to determine the best relay locations and power allocation scheme. By adding a cooperative relay in optimum location, the network lifetime increases 3 times over the noncooperative WSN.

\section{REFERENCES}

[1] J.-H. Chang and L. Tassiulas, "Maximum lifetime routing in wireless sensor networks," IEEE/ACM Trans. on Networking, vol. 12, no. 4, pp. 609-619, Aug. 2004.

[2] I. Kang and R. Poovendran, "Maximizing static network lifetime of wireless broadcast adhoc networks," Proc. of ICC'03, May 2003.

[3] M. Bhardwaj, T. Garnett, and A. P. Chandrakasan, "Upper bounds on the lifetime of sensor networks," Proc. of the Int. Conf. on Commun., 2001.

[4] R. J. M. II, A. K. Das, and M. El-Sharkawi, "Maximizing lifetime in an energy constrained wireless sensor array using team optimization of cooperating systems," Proc. of the Int. Joint Conf. on Neural Networks, May 2002.

[5] P. Floreen, P. Kaski, J. Kohonen, and P. Orponen, "Multicast time maximization in energy constrained wireless networks," Proc. of ACM/IEEE Mobicom 2003 Workshop on Foundations of Mobile Computing, Sept. 2003.

[6] I. Maric and R. D. Yates, "Cooperative broadcast for maximum network lifetime," Conf. on Inform. Sciences and Systems, vol. 1, pp. 591-596, 2004.

[7] I. Maric and R. D. Yates, "Cooperative multicast for maximum network lifetime," IEEE JSAC, vol 23, pp. 127-135, Jan. 2005.

[8] J. N. Laneman and G. W. Wornell, "Distributed space-time coded protocols for exploiting cooperative diversity in wireless networks," IEEE Trans. on Inform. Theory, vol. 49, pp. 2415-2525, Oct. 2003.

[9] J. N. Laneman, D. N. C. Tse, and G. W. Wornell, "Cooperative diversity in wireless networks: efficient protocols and outage behavior," IEEE Trans. on Inform. Theory, vol. 50, pp. 3062-3080, Dec. 2004.

[10] W. Su, A. K. Sadek, and K. J. R. Liu, "SER performance analysis and optimum power allocation for decode-and-forward cooperation protocol in wireless networks," in Proc. WCNC 2005, vol. 2, pp. 984 - 989, Mar. 2005.

[11] J. Luo, R. S. Blum, L. J. Greenstein, L. J. Cimini, and A. M. Haimovich, "New approaches for cooperative use of multiple antennas in ad hoc wireless networks," Proc. IEEE Vehicular Technology Conf., vol. 4, pp. 2769-2773, Sep. 2004

[12] H. Zhu, T. Himsoon, W. P. Siriwongpairat, and K. J. R. Liu, "Energy-efficient cooperative transmission over multiuser OFDM networks: who helps whom and how to cooperate," Proc. IEEE WCNC 2005, vol. 2, pp. 1030-1035, Mar. 2005.

[13] C.-M. Cheng and H. T. Kung, "Use of relays in extending network lifetime," IEEE Vehicular Tech. Conf., vol. 7, pp. 4655-4659, Sep. 2004.

[14] R. A. Horn and C. R. Johnson, Matrix Analysis, Cambridge Univ. Press, New York, 1985. 